\title{
LIXIVIAÇÃO DE GLYPHOSATE E IMAZAPYR EM SOLOS COM DIFERENTES TEXTURAS E COMPOSIÇÃO QUÍMICA. I - MÉTODO DO BIOENSAIO ${ }^{1}$
}

\author{
ADAILSON P. de SOUZA ${ }^{2}$, FRANCISCO A. FERREIRA ${ }^{3}$, ANTÔNIO A. da SILVA ${ }^{3}$, HUGO A. RUIZ ${ }^{4}$ e HÉLIO T. PRATES
}

\section{RESUMO}

A mobilidade dos herbicidas no perfil do solo é influenciada por vários processos, tais como, retenção, transformação e transporte. O conhecimento destes fenômenos é fundamental para a perfeita compreensão do destino de tais produtos no ambiente. Dentre as várias técnicas utilizadas nesses estudos, o método do bioensaio apresenta-se como de ótima representatividade e reprodutibilidade. Em razão dessas características, associadas a poucas informações sobre a mobilidade de herbicidas nos solos sob condições tropicais, foi conduzido um bioensaio objetivando verificar o movimento vertical do glyphosate e do imazapyr, em colunas de solos de diferentes texturas e composição química, utilizando-se o tomateiro (Lycopersicon esculentum Mill var. Santa Clara) como planta-teste. Os resultados desse estudo permitiram concluir que: a) o limite de detecção do bioensaio para o glyphosate e para o imazapyr corresponde ao menor valor de $\mathrm{I}_{50}$ obtido na curva padrão, 331,52 e 5,4 $\mu \mathrm{g} \mathrm{L}^{-1}$, respectivamente; b) as concentrações do glyphosate biologicamente ativo nos lixiviados dos solos de Viçosa e de Sabará encontram-se abaixo do limite de detecção do bioensaio; c) o glyphosate apresentou, na coluna de $1 \mathrm{~cm}$, mobilidade muito baixa nos solos estudados; d) a mobilidade do imazapyr, na coluna de $30 \mathrm{~cm}$, é maior no solo de textura francoarenosa de Viçosa; e) o alto teor de argila e de matéria orgânica do solo de Sabará apresentam-se como os principais fatores de retenção do imazapyr nesse solo.

Palavras chave: Logística, dose-resposta, herbicida.

\section{ABSTRACT}

\section{Glyphosate and imazapyr leaching in soils with different textures and chemical composition. I - Bioassay method}

The herbicide mobility in the soil profile is influenced by several processes: retention, transformation and transport. This phenomenon knowledge is essential to the perfect comprehension of these product destines in the environment. Among the several techniques used in such studies, the bioassay method presents optimum representativeness. Because these characteristics and the scarce information about the herbicide mobility in soils under tropical conditions, a bioassay was carried out aiming to verify the vertical movement of glyphosate and imazapyr in soil columns with different textures and chemical composition using the tomato (Lycopersicon esculentum Mill var. Santa Clara) as test-plant. According to the results it may be concluded that: a) the bioassay detection boundary for both the glyphosate and imazapyr corresponds

\footnotetext{
${ }^{1}$ Recebido para publicação em 28/05/98 e na forma revisada em 13/08/99.

${ }^{2}$ Prof $^{\mathrm{o}}$ Adjunto do Dept ${ }^{\mathrm{o}}$ de Solos e Eng. Rural da UFPB, CEP: $58397-000$, Areia/PB.

${ }^{3}$ Prof $^{\circ}$ Adjunto do Dept ${ }^{\circ}$ de Fitotecnia da UFV, CEP: 36571-000, Viçosa/MG.

${ }^{4}$ Prof $^{\text {o }}$ Adjunto do Dept ${ }^{\circ}$ de Solos da UFV, CEP: $36571-000$, Viçosa/MG.

${ }^{5}$ EMBRAPA/CNPMS, C.P. 151, CEP: 35701-970, Sete Lagoas/MG.
} 
to the lowest $I_{50}$ value obtained in the pattern curve that is 331.52 and $5.4 \mu \mathrm{g} \mathrm{L}^{-1}$ respectively; b) the concentrations of the biologically active glyphosate in the leaching products of Viçosa and Sabará soils are bellow the bioassay detection boundary; c) in the $1 \mathrm{~cm}$ column, the glyphosate presented a very low mobility in the studied soils;

\section{INTRODUÇÃO}

No último quarto do século $\mathrm{XX}$, o aumento na síntese de novos herbicidas pelas indústrias tem levado os pesquisadores a uma busca constante de respostas que ajudem a compreender o comportamento desses compostos no ambiente. As propriedades físicas e químicas dos herbicidas, os métodos de aplicação, as características bióticas e abióticas do ambiente e as condições climáticas influenciam grandemente em sua atividade. No entanto, deve-se ressaltar que a relação de causa e efeito entre o herbicida usado na agricultura, ou outras atividades nãoagrícolas, e a observação da contaminação do ambiente podem não ser, em muitos casos, facilmente estabelecidos. Como esta relação está geralmente associada à presença do produto no ambiente edáfico, torna-se essencial que os processos que influenciam o seu transporte no solo sejam perfeitamente entendidos antes que qualquer relação de causa e efeito seja estabelecida.

O comportamento de um herbicida no solo é influenciado por processos de retenção, transformação e transporte que ocorrem imediatamente e/ou, simultaneamente, após a sua aplicação (Weber \& Miller, 1989). Os trabalhos de pesquisa visam esclarecer a natureza e a intensidade desses processos, buscando encontrar meios capazes de predizer o comportamento desses produtos no ambiente.

A mobilidade do herbicida no solo, a qual influencia diretamente o seu desempenho no controle de plantas daninhas e a sua dissipação no ambiente, pode ser avaliada por diferentes métodos, sendo os mais comuns: lisímetro (Edwards \& Glass, 1971; Ogner, 1987; Winton \& d) in the $30 \mathrm{~cm}$ column, the imazapyr mobility is greater in the sandy loam texture soil of Viçosa; e) the high clay and organic matter contents in Sabará soil present as the main retention factors of imazapyr in this soil. herbicide.

Key words: Logistic, dose-response, Weber, 1996); deslocamento miscível (Elrick \& French, 1966); cromatografia de camada delgada de solo (Hilling, 1971; Sprankle et al., 1975a; Sánchez-Martín et al., 1994); coluna de lixiviação (Weber, 1972; Weber et al., 1986; Günther et al., 1993; Krzyszowska et al., 1994). O método mais adequado é aquele que melhor se aproxima das condições de campo e apresenta uma boa reprodutibilidade. Em razão dessas características, o método da coluna de lixiviação tem sido largamente utilizado nos ensaios com o glyphosate (Cheah et al., 1997) e com as imidazolinonas (Liu \& Weber, 1985; McKinnon \& Weber, 1988; Mangels, 1991).

Parte do herbicida que atinge o solo pode ser transportado para o seu interior pela água de chuva ou de irrigação, em duas etapas. Inicialmente, o herbicida e a água movem-se sob uma condição saturada. Em seguida, inicia-se um fluxo sob uma condição insaturada. Em ambos os casos, a atuação de forças como a difusão no interior dos agregados, a ação da capilaridade promovida pela evaporação da água na superfície do solo e a adsorção pelos colóides do solo, agem diretamente sobre o herbicida na solução. Segundo Clay (1993), o herbicida pode alcançar elevadas profundidades do solo ou ainda o lençol freático. A molécula do herbicida, a intensidade das precipitações e fatores sazonais influenciam o processo. A possibilidade de movimento ascendente do herbicida lixiviado do subsolo deve também ser considerada. Isso se torna mais evidente no caso dos herbicidas fracamente adsorvidos, sob condições de elevada evapotranspiração, tal como algumas imidazolinonas (Mangels, 1991).

Os atributos do solo que mais influenciam o movimento descendente dos herbicidas são: o 
conteúdo e o tipo de matéria orgânica, a composição e a distribuição do tamanho das partículas do solo, o $\mathrm{pH}$, a densidade aparente, e o tamanho e a distribuição dos poros. Para os herbicidas de elevada capacidade adsortiva, o conteúdo de matéria orgânica é geralmente o fator mais importante, pois, quanto mais alto menor a lixiviação (Sprankle et al., 1975b; Madhun et al., 1986; Senesi et al., 1994). A lixiviação será ainda maior em solos de textura arenosa que em solos siltosos ou argilosos (Nicholls, 1988). Para o glyphosate e algumas imidazolinonas, o tipo de argila, o conteúdo de óxidos e o pH influenciam na sua adsorção e, conseqüentemente, na sua lixiviação (Wehtje et al., 1987; Carlisle \& Trevors, 1988).

Em decorrência da grande variabilidade das características físicas, químicas e biológicas dos solos, espera-se que a natureza e a intensidade dos processos de lixiviação desses produtos sejam também diferentes. A avaliação dessas características, no comportamento dos herbicidas, define, na prática, a escolha dos herbicidas com menor potencial de lixiviação ou a variação das doses, quando aplicados a solos distintos.

Diante do exposto, o principal objetivo do presente trabalho foi estudar o efeito residual dos herbicidas glyphosate e imazapyr, verificando o seu movimento vertical em colunas de solo.

\section{MATERIAL E MÉTODOS}

$\mathrm{O}$ experimento foi conduzido no Laboratório de Herbicida no Solo e em casa de vegetação do Departamento de Fitotecnia da Universidade Federal de Viçosa, MG, com amostras de solo provenientes das subestações da CEMIG, dos municípios de Viçosa-MG e SabaráMG. As amostras foram coletadas na camada superficial do solo (de 0 a $20 \mathrm{~cm}$ ), secas ao ar e passadas em peneiras de 4,0 e 2,0 $\mathrm{mm}$ de abertura de malha, para os ensaios de lixiviação e caracterização química, física e mineralógica (Tabela 1), respectivamente.

Os herbicidas utilizados foram $\mathrm{o}$ glyphosate e o imazapyr (Figura 1), que são inibidores da síntese de aminoácidos. O glyphosate (Figura 1a) é não-seletivo, de aplicação em pós-emergência das plantas daninhas, com absorção foliar e rápida translocação pelo simplasto da planta (Monsanto, 1990). O imazapyr (Figura 1b) é também um herbicida sistêmico, de amplo espectro aplicado em pósemergência, com ação pré-emergente residual, recomendado para vegetação infestante em áreas não-cultivadas. Controla plantas daninhas, mono e dicotiledôneas anuais e perenes (Rodrigues \& Almeida, 1995).

TABELA 1. Composição química, física e mineralógica das amostras dos solos.

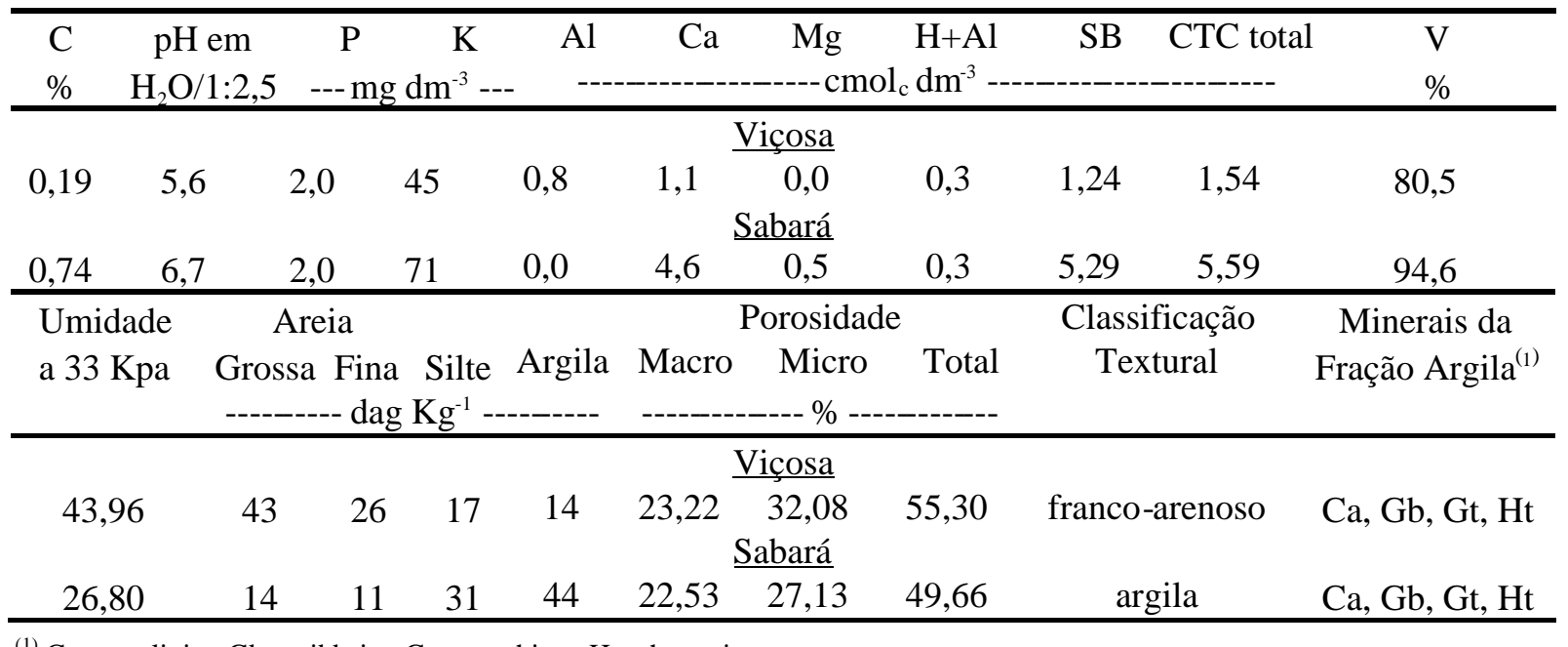

${ }^{(1)} \mathrm{Ca}=$ caulinita, $\mathrm{Gb}=$ gibbsita, $\mathrm{Gt}=$ goethita e $\mathrm{Ht}=$ hematita. 
a<smiles></smiles>

Sal de isopropilamino de N-(fosfonometil)-glicina

b<smiles>CC(C)C1(C)N=C(c2ncccc2C(=O)O)NC1=O</smiles>

Ácido 2-(4-isopropil-4-metil-5-oxo-2-imidazolina-2-ilo) nicotínico

FIGURA 1. Fórmulas estruturais do glyphosate (a) e do imazapyr (b).

Para estudar o movimento dos herbicidas no solo, foram utilizados anéis de tubos de PVC com 1 e $5 \mathrm{~cm}$ de altura e $9,8 \mathrm{~cm}$ de diâmetro interno. As colunas com as alturas de $1 ; 5 ; 10 ; 15$ e $30 \mathrm{~cm}$, formadas pela junção dos anéis, tiveram as suas paredes internas previamente parafinadas, antes de receberem o solo, a fim de promover a formação de uma camada hidrofóbica, evitandose, assim, a possível formação de um fluxo de água pelas paredes das mesmas. Na parte inferior das colunas, foram colocados discos de papelfiltro de filtragem rápida (INLAB - $\mathrm{n}^{\mathrm{Q}} 10$ porosidade de $10 \mu \mathrm{m})$ de $11 \mathrm{~cm}$ de diâmetro e uma malha de gaze (abertura de malha de $1 \mathrm{~mm}$ ), presa por elástico, objetivando reter o solo na coluna, deixando, entretanto, passagem para o lixiviado (Figura 2).

Nas colunas foram colocados os solos em quantidade suficiente para alcançar a altura desejada $(1 ; 5 ; 10 ; 15$ e $30 \mathrm{~cm})$ e levadas para uma mesa vibratória até ser obtida uma densidade aparente de $1,2 \mathrm{~g} \mathrm{~cm}^{-3}$. Em seguida, as colunas contendo os materiais de solo foram colocadas em bandejas com água destilada durante um período suficiente para que houvesse a saturação por capilaridade. Após esse período, o excesso de água foi drenado, colocando-se as colunas sobre béqueres, por um período de 24 a 48 h, tempo suficiente para que a umidade do solo ficasse próxima à capacidade de campo $(33 \mathrm{kPa})$. Em seguida, foi feita a aplicação do herbicida, uniformemente, por meio de uma seringa de $5 \mathrm{ml}$, com a extremidade da agulha em forma de leque. A quantidade de herbicida aplicada correspondeu à dose de $4 \mathrm{~L} \mathrm{ha}^{-1}$ do produto comercial Roundup e Arsenal (480 $\mathrm{g} \mathrm{L}^{-1}$ de sal de glyphosate e $266,3 \mathrm{~g}$ $\mathrm{L}^{-1}$ de imazapyr, respectivamente) em uma calda de 400 L. Após a aplicação dos produtos, as colunas foram colocadas sobre recipientes de captação e levadas para um simulador de chuva, onde foram submetidas a um regime hídrico de 40 $\mathrm{mm} \mathrm{h}^{-1}$ por um período de $4 \mathrm{~h}$, tempo suficiente para se obter um volume de $950 \mathrm{ml}$ de lixiviado por coluna. 

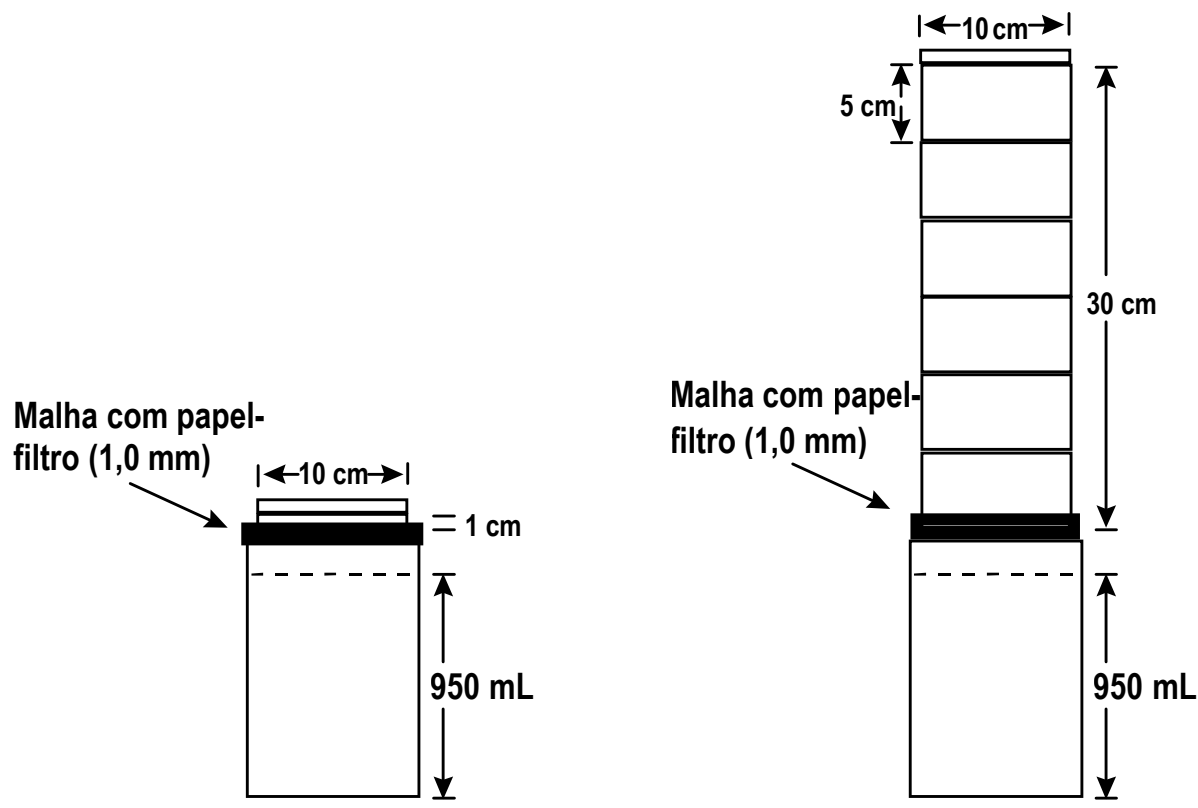

FIGURA 2. Representação esquemática das colunas usadas no estudo da lixiviação dos herbicidas glyphosate $(1,0 \mathrm{~cm})$ e imazapyr $(30 \mathrm{~cm})$.

Doses crescentes do lixiviado $(0 ; 0,093$; 0,$1875 ; 0,375 ; 0,75$ e $1,5 \mathrm{~L}$, correspondentes a 0 ; 6,$25 ; 12,5 ; 25 ; 50$ e $100 \%$ do volume de solução para o glyphosate e $0 ; 0,0075 ; 0,015 ; 0,03 ; 0,06$; e $0,12 \mathrm{~L}$, correspondentes a $0 ; 0,5 ; 1 ; 2 ; 4 ;$ e $8 \%$ do volume de solução para o imazapyr), foram colocadas em vasos de $2 \mathrm{~L}$, revestidos com sacos de polietileno e, em seguida, completou-se o volume de 1,5 $\mathrm{L}$ com água. Adicionaram-se, em cada vaso, $1,5 \mathrm{ml} \mathrm{de} \mathrm{MgSO}_{4} \cdot 7 \mathrm{H}_{2} 01 \mathrm{M} ; 1,5 \mathrm{ml}$ de $\mathrm{KH}_{2} \mathrm{PO}_{4} 1 \mathrm{M} ; 7,5 \mathrm{ml}$ de $\mathrm{KNO}_{3} 1 \mathrm{M} ; 7,5 \mathrm{ml}$ de $\mathrm{Ca}\left(\mathrm{NO}_{3}\right)_{2} \cdot 4 \mathrm{H}_{2} \mathrm{O} 1 \mathrm{M} ; 1$ mlde FeEDTA e $1 \mathrm{ml}$ de micro-Fe, para dar condições de crescimento à planta-teste (Lycopersicon esculentum Mill var. Santa Clara). Em seguida, duas mudas das plantasteste (idade de cinco dias após germinadas em areia de quartzo) foram transplantadas para cada vaso. Os vasos foram mantidos sob aeração constante e o pH da solução corrigido diariamente para 5,7 $\pm 0,02$. Vinte dias após, efetuou-se a colheita das plantas, determinando-se $o$ comprimento das raízes. Após a separação da parte aérea das raízes, as mesmas foram acondicionadas separadamente em sacos de papel e colocadas para secar em estufa com circulação de ar a $65^{\circ} \mathrm{C}$, até peso constante. Após a secagem do material (aproximadamente $72 \mathrm{~h}$ ) este foi pesado e analisado estatisticamente.

O delineamento estatístico utilizado para cada herbicida foi o inteiramente casualizado, com os tratamentos dispostos em um esquema fatorial $2 \times 6$ com três repetições, sendo que o primeiro fator correspondeu aos solos (Viçosa e Sabará) e o segundo às doses do lixiviado.

Simultaneamente ao ensaio de lixiviação, foi realizado um experimento em solução nutritiva para determinar, com base na curva-padrão do bioindicador, a dose dos herbicidas que reduziria em $50 \%$ o desenvolvimento da planta-teste $\left(I_{50}\right)$. O delineamento estatístico para cada herbicida foi o inteiramente casualizado, com seis tratamentos (doses crescentes dos herbicidas glyphosate, 0; 32; 64; $128 ; 256$ e $512 \mu \mathrm{g} \mathrm{L}^{-1}$ e imazapyr, $0 ; 1,775$; 3,$55 ; 7,1 ; 14,2$ e $\left.28,4 \mu \mathrm{g} \mathrm{L}^{-1}\right)$ e cinco repetições.

As características avaliadas, por planta, em ambos os experimentos (biomassa seca total, BST, da parte aérea, BSA, das raízes, BSR e comprimento das raízes, CR), permitiram estimar uma variância ponderada entre a variância experimental e a de amostragem. A relação da 
BST, da BSA, da BSR e do CR com as doses do herbicida e do lixiviado foi analisada por regressão, testando-se o modelo logístico. Tanto para a análise de variância quanto para a estimativa dos parâmetros da regressão do modelo, utilizou-se o pacote estatístico SAEG (SAEG, 1997).

A concentração dos herbicidas no lixiviado foi obtida mediante o uso da fórmula:

$$
C C=\frac{I_{50 P} \times V_{L x}}{\frac{I_{50 L x}}{100} \times V_{P}},
$$

em que,

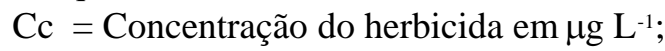

$\mathrm{I}_{50 \mathrm{P}}=\mathrm{I}_{50}$ da curva padrão;

$\mathrm{I}_{50 \mathrm{Lx}}=\mathrm{I}_{50}$ da curva do lixiviado;

$\mathrm{V}_{\mathrm{Lx}}=$ Volume de solução no vaso da curva do

lixiviado; e

$V_{P}=$ Volume de solução no vaso da curva padrão.

\section{RESULTADOS E DISCUSSÃO}

Observa-se pela Figura 3 que a função logística apresentou, nas diferentes variáveis analisadas, ótimo ajustamento para os herbicidas $\left(\mathrm{R}^{2}>0,9\right)$. Constata-se, ainda, que o tomateiro apresenta elevada sensibilidade aos herbicidas estudados, sendo este mais sensível ao imazapyr, tendo sido encontrados os menores valores de $\mathrm{I}_{50}$, e a variável BSR é a que melhor responde ao ajuste da função logística $\left(R^{2}=0,9919\right)$, com o menor valor de $\mathrm{I}_{50}\left(5,4 \mu \mathrm{g} \mathrm{L}^{-1}\right)$. Ambos os herbicidas apresentam mecanismos de ação semelhantes, e a diferença entre os valores de $\mathrm{I}_{50}$ pode estar relacionada à maior ou à menor capacidade de absorção e translocação via sistema radicular. Uma vez absorvido pelas raízes, esses herbicidas apresentam mobilidade normalmente maior no apoplasto que no simplasto; a evapotranspiração na superfície das folhas arrasta a água e seus solutos para as raízes, mantendo alto gradiente de concentração dessas moléculas entre a solução nutritiva e o apoplasto da raiz. Quando a planta transpira, o fluxo desses herbicidas para o xilema da raiz é determinado pela sua passagem em uma camada de células (estrias de Caspary) cujas paredes anticlinais são impermeáveis a água e solutos. Segundo Little \& Shaner (1991), o imazapyr é facilmente absorvido pelo sistema radicular, sendo rapidamente translocado via xilema e retranslocado via floema para toda a planta. Essa maior absorção e translocação ocorrem em razão de as propriedades lipofílicas do imazapyr serem mais favoráveis à sua permeabilidade pela membrana das células das estrias de Caspary.

A concentração dos herbicidas no lixiviado (eq.1) é uma função dos valores de $\mathrm{I}_{50}$ obtidos na curva-padrão (Figura 3 ) e da curva do lixiviado (Figuras 4 e 5). De acordo com a Figura 4, observa-se que não houve o efeito da percentagem de lixiviado dos solos de Viçosa e de Sabará, tratados com glyphosate, sobre as variáveis biomassa seca e comprimento de raiz, nas diferentes características avaliadas. Esses resultados demonstram que a concentração do glyphosate biologicamente ativo no lixiviado encontra-se abaixo da capacidade de determinação do $I_{50}$ pela planta bioindicadora. Essa baixa concentração no lixiviado reflete sua elevada retenção pela camada de $1,0 \mathrm{~cm}$ de solo ou, ainda, o mesmo se encontraria em uma forma inativa na solução, seja por complexos formados pela sua ligação com íons metálicos e/ou, compostos orgânicos, seja por ligações químicas à superfície das argilas em solução (Hensley et al., 1978). Em ambos os casos, é confirmada a alta capacidade de inativação do glyphosate pelos constituintes do solo, onde as argilas (Sprankle et al., 1975a; Glass, 1987) e a matéria orgânica (Sprankle et al., 1975b; Piccolo et al., 1996) agem intensamente nesse processo. 


\section{Glyphosate}
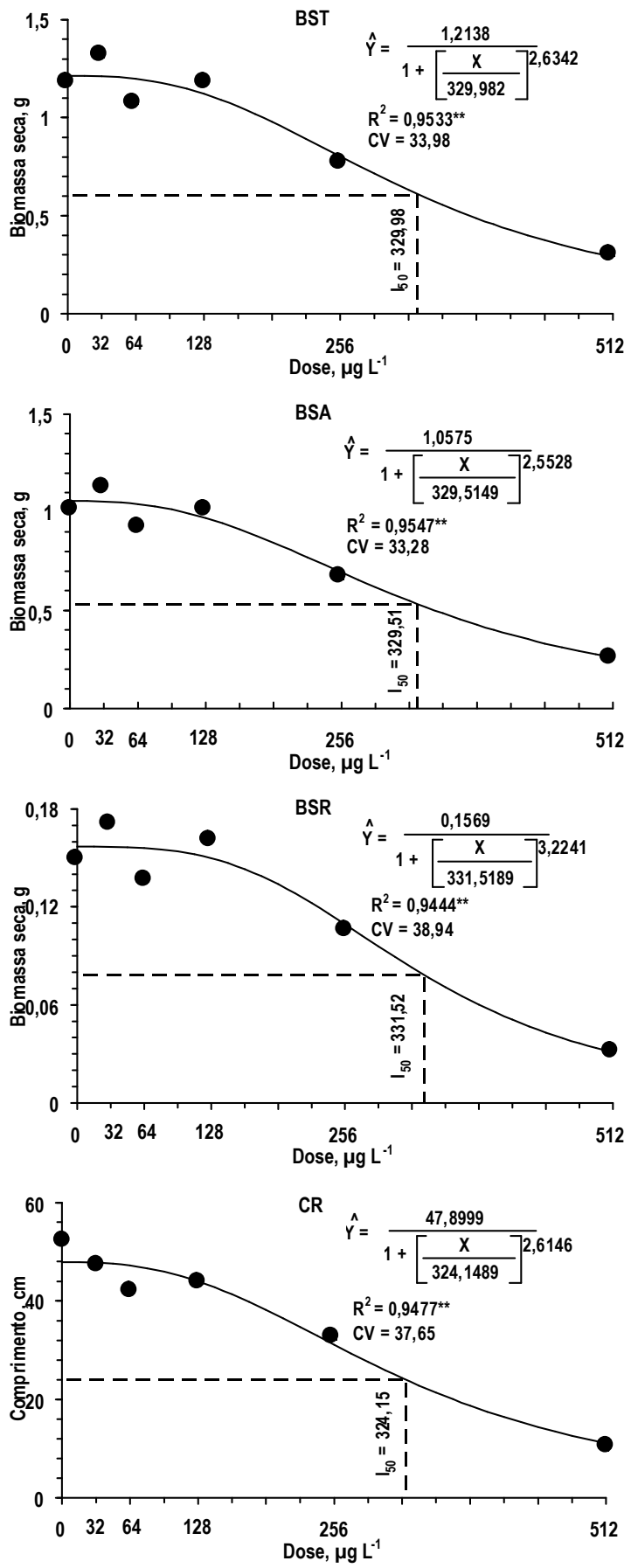

\section{Imazapyr}

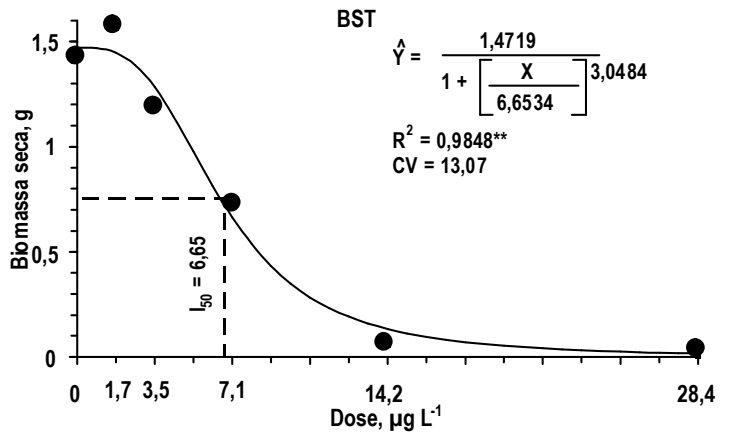

BSA

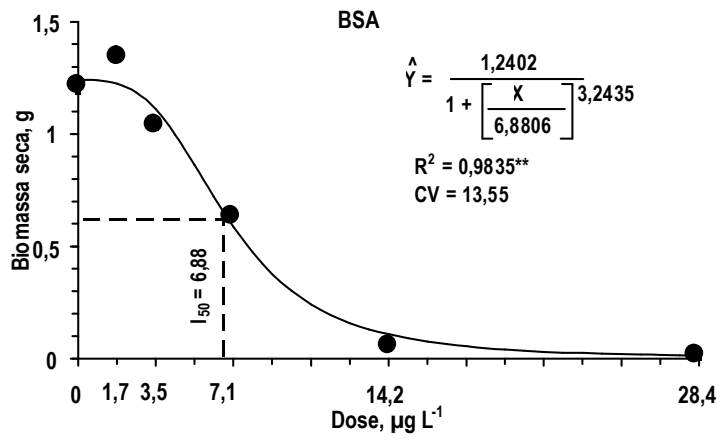

BSR

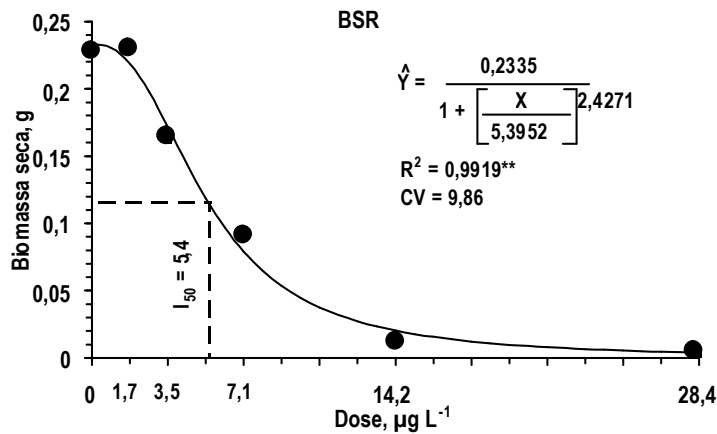

CR

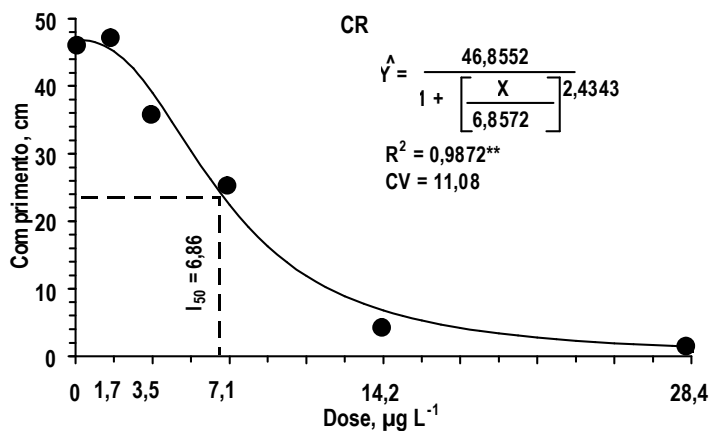

FIGURA 3. Biomassa seca total, BST, da parte aérea, BSA, das raízes, BSR, e comprimento das raízes, $\mathrm{CR}$, do tomateiro (Lycopersicon esculentum Mill var. Santa Clara), em resposta a diferentes doses do glyphosate e do imazapyr, em cultivo hidropônico. 
Viçosa

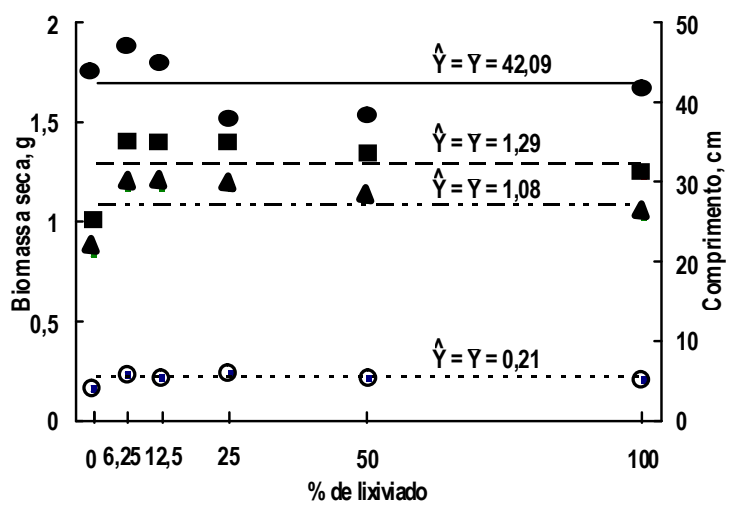

Sabará

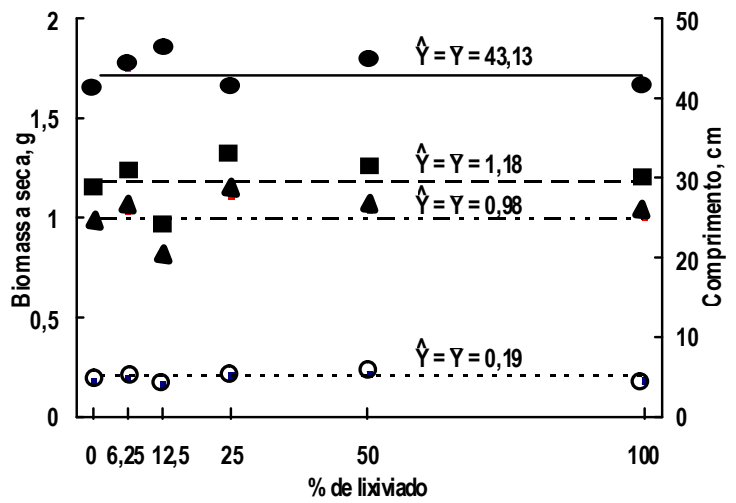

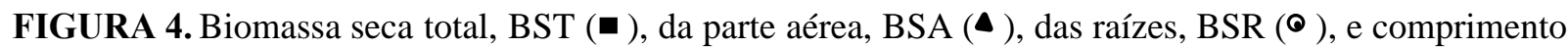
das raízes, CR $(\bullet)$ do tomateiro (Lycopersicon esculentum var. Santa Clara), em resposta a dosagem de diferentes quantidades de lixiviado de dois solos (Viçosa e Sabará), após aplicação de glyphosate, em colunas de $1,0 \mathrm{~cm}$.

Na Figura 5, observa-se a resposta do tomateiro à dosagem de diferentes quantidades de lixiviado dos solos de Viçosa e Sabará, após a aplicação do imazapyr nas colunas de solo. Os altos valores de $\mathrm{R}^{2}(>0,95)$ demonstram o bom ajuste da função logística aos valores observados nas diferentes características avaliadas. Verificase, ainda, que o lixiviado do solo de Viçosa apresentou os menores valores de $\mathrm{I}_{50}$, para todas as características avaliadas, onde para a BSR foi observado o menor $I_{50}(1,65 \%)$. No lixiviado do solo de Sabará, o menor valor foi encontrado no CR $(3,57 \%)$.

De acordo com a Tabela 2, verifica-se que o glyphosate encontra-se em concentrações menores que os valores estimados pela curvapadrão (Figura 3), não sendo possível estimar a concentração real do produto no lixiviado da coluna de $1,0 \mathrm{~cm}$. A impossibilidade da estimativa desse valor deu-se em razão da resposta não significativa do bioindicador às diferentes dosagens do lixiviado (Figura 4). Tais resultados demonstram a baixa mobilidade do glyphosate nos solos de Viçosa e de Sabará. Essa baixa mobilidade ocorreu, provavelmente, em razão das ligações atribuídas ao grupo fosfônico (Figura 1), reagindo com cátions polivalentes adsorvidos às argilas, bem como a matéria orgânica desses solos
(Tabela 1). Resultados semelhantes foram obtidos por Sundaram \& Sundaram (1997) que, ao estudarem a influência dos produtos de solubilidade de seis complexos glyphosate-íons metálicos, no solo e em areia, verificaram, em bioensaio com tomateiro, que as fortes ligações do glyphosate com os íons $\mathrm{Mg}, \mathrm{Ca}, \mathrm{Mn}, \mathrm{Zn}, \mathrm{Cu}$ e Fe, reduziram sua toxicidade.

De acordo com os resultados apresentados para o imazapyr (Tabela 2), verifica-se que o lixiviado da coluna de $30 \mathrm{~cm}$ do solo de Viçosa apresentou, nas diferentes características avaliadas, as mais altas concentrações com uma variação de 322,6 a $355,44 \mu \mathrm{g} \mathrm{L} \mathrm{L}^{-1}$, enquanto que no lixiviado do solo de Sabará a variação foi de 139,54 a $191,96 \mu \mathrm{g} \quad \mathrm{L}^{-1}$. A diferença de concentração nos dois lixiviados, que em média foi de 339,44 e $165,19 \mu \mathrm{g} \mathrm{L}^{-1}$, para o solo de Viçosa e Sabará, respectivamente, pode ser atribuída às diferenças químicas e físicas dos solos. O imazapyr (Figura 1) apresenta a propriedade de um ácido fraco, o que torna sua ligação com os constituintes do solo de fraca intensidade e fortemente influenciada pelas variações de $\mathrm{pH}$ do solo (Dickens \& Wehtje, 1986). Mangels (1991) afirma que o $\mathrm{pH}$ e a matéria orgânica influenciam acentuadamente o movimento do imazapyr no solo. 
Viçosa
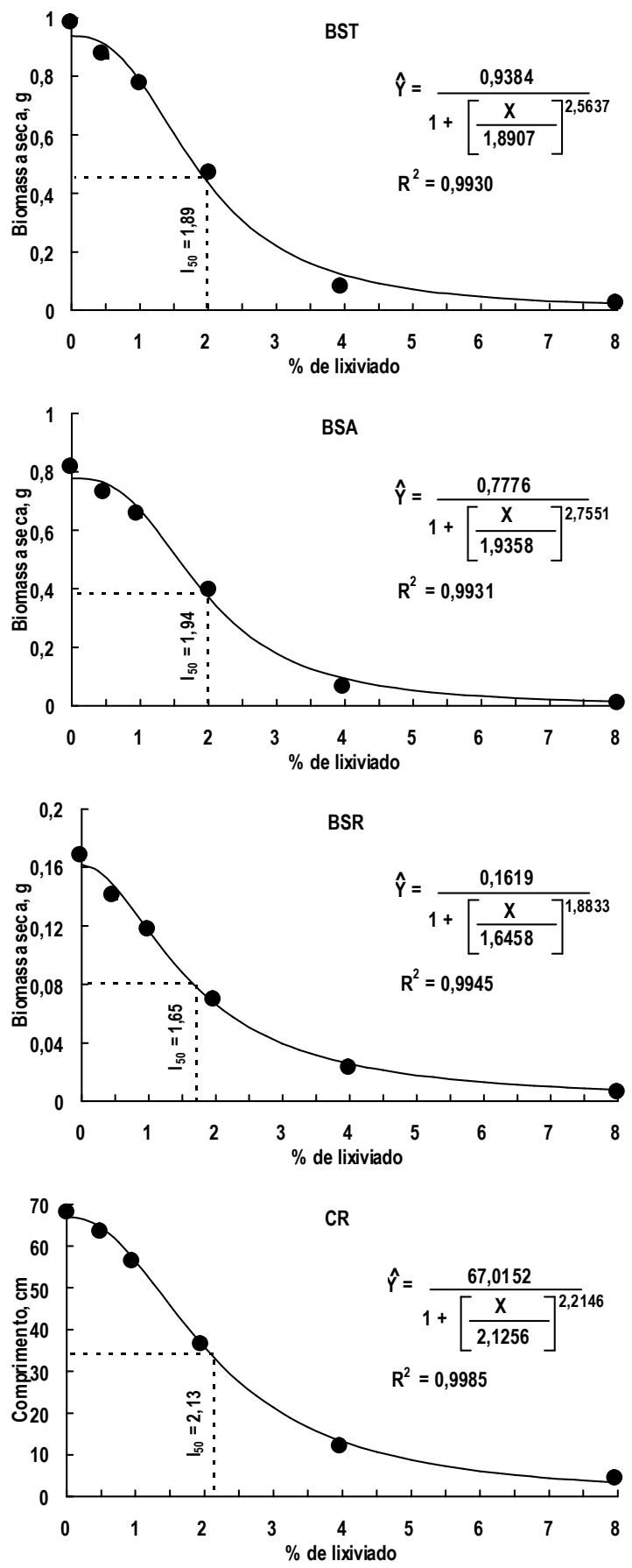

Sabará
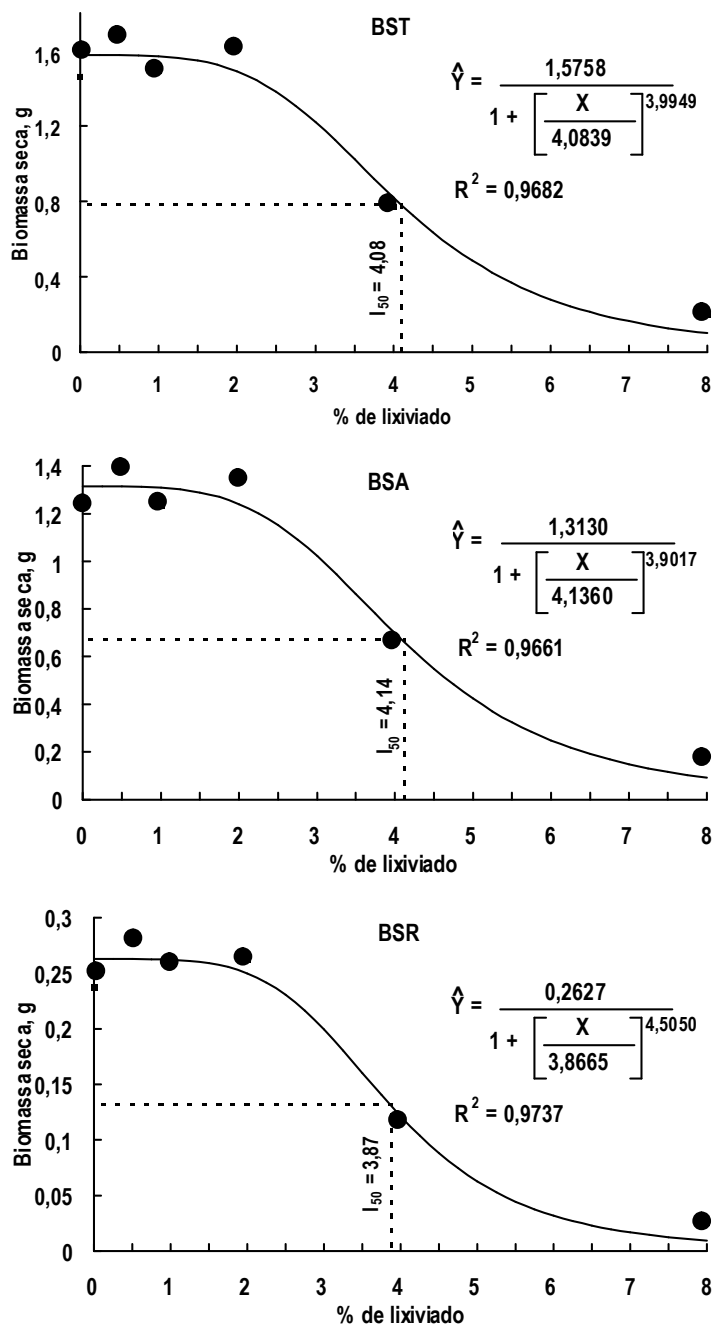

CR

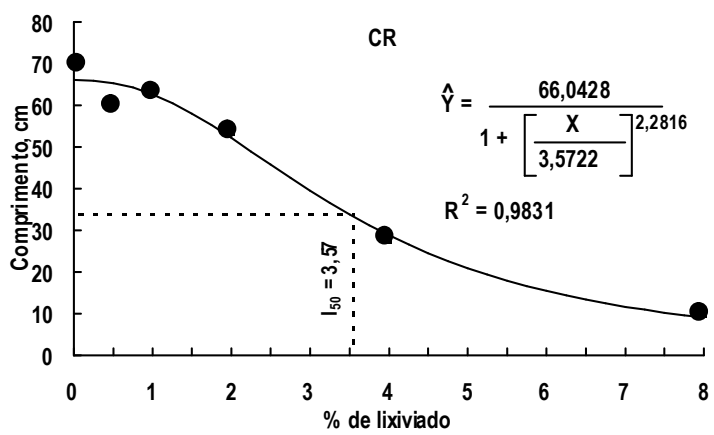

FIGURA 5. Biomassa seca total, BST, da parte aérea, BSA, das raízes, BSR, e comprimento das raízes, CR, do tomateiro (Lycopersicon esculentum Mill var. Santa Clara), em resposta a dosagem de diferentes quantidades de lixiviado de dois solos (Viçosa e Sabará), após aplicação de imazapyr, em colunas de $30 \mathrm{~cm}$. 
TABELA 2. Concentração dos herbicidas (eq. 1) nos lixiviados dos solos de Viçosa e de Sabará, em função do $\mathrm{I}_{50}$ para biomassa seca total, BST, parte aérea, BSA, raízes, BSR, e comprimento das raízes, CR, do tomateiro (Lycopersicon esculentum Mill var. Santa Clara).

\begin{tabular}{|c|c|c|c|c|}
\hline Solo & BST & BSA & $\overline{B S R}$ & $\overline{\mathrm{CR}}$ \\
\hline & \multicolumn{4}{|c|}{ Glyphosate, $\mu \mathrm{g} \mathrm{L}^{-1}$} \\
\hline Viçosa & $<329,98$ & $<329,51$ & $<331,52$ & $<324,15$ \\
\hline \multirow[t]{2}{*}{ Sabará } & $<329,98$ & $<329,51$ & $<331,52$ & $<324,15$ \\
\hline & \multicolumn{4}{|c|}{ Imazapyr, $\mu \mathrm{g} \mathrm{L}^{-1}$} \\
\hline Viçosa & 351,90 & 355,44 & 327,82 & 322,60 \\
\hline Sabará & 162,92 & 166,35 & 139,54 & 191,96 \\
\hline
\end{tabular}

Segundo Nicholls (1988), a adsorção no solo de moléculas orgânicas de caráter ácido fraco ocorreria predominantemente por forças de van der Walls e interação dipolo-dipolo. Sendo os minerais de argila que predominam nesses solos de baixa atividade, acredita-se que a adsorção do imazapyr ocorre com maior intensidade pela matéria orgânica, o que justificaria sua menor concentração no lixiviado do solo de Sabará, cujo teor de matéria orgânica é maior $(0,74 \%$ C) (Tabela 1).

\section{LITERATURA CITADA}

CARLISLE, S.M., TREVORS, J.T. Glyphosate in the environment. Water air soil pollut., n.39, p.409-420, 1988.

CHEAH, U., KIRKWOOD, R.C., LUM, K. Adsorption, desorption and mobility of four commonly used pesticides in Malaysian agricultural soils. Pestic. sci., n.50, p.53-63, 1997.

CLAY, D.V. Herbicide residues in soils and plants and their bioassay. In: STREIBIG, J.C., KUDSK, P. Herbicide bioassays. Florida: CRC Press, Inc, 1993. p.153-171.
DICKENS, R., WEHTJE, G. Mobility and soil solution characteristics of imazapyr (Arsenal) and sulfometuron methyl (Oust) in Alabama soils. Proc. So. Weed Sci. Soc. Am., n.39, p.368, 1986.

EDWARDS, W.M., GLASS, B.L. Methoxychlor and 2,4,5- $\mathrm{T}$ in lysimeter percolation and runoff water. Bull. environ. contam. toxicol., n.6, p.81-84, 1971.

ELRICK, D.E., FRENCH, L.K. Miscible displacement patterns on disturbed and undisturbed soil cores. Soil Sci. Soc. Am. Proc., n.30, p.153-156, 1966.

GLASS, R.L. Adsorption of glyphosate by soils and clay minerals. J. agric. food chem., v.35, n.4, p.497-500, 1987.

GÜNTHER, P., PESTEMER, W., RAHMAN, A., NORDMEYER, H. A bioassay technique to study the leaching behaviour of sulfonylurea herbicides in different soil. Weed Res., n.33, p.177-185, 1993.

HENSLEY, D.L., BEUERMAN, D.S.N., CARPENTER, P.L. The inactivation of glyphosate by various soil and metal salts. Weed Res., n.18, p.287, 1978. 
HILLING, C.E. Pesticide mobility in soil. II. Applications of soil thin-layer chromatography. Soil Sci. Soc. Am. Proc., n.35, p.562-563, 1971.

KRZYSZOWSKA, A.J., ALLEN, R.D., VANCE, G.F. Assessment of the fate of two herbicides in a Wyoming rangeland soil: column studies. J. environ. qual., n.23, p.1051-1058, 1994.

LITTLE, D.L., SHANER, D.L. Absorption and translocation of the imidazolinone herbicides. In: SHANER, D.L., O'CONNOR, S.L. (Eds.). The imidazolinone herbicides, Florida: CRC Press, Inc., 1991. p.53-69.

LIU, S.L., WEBER, J.B. Retention and mobility of AC 252,214, chlorsulfuron, prometryn and SD95481 in soil. Proc. So. Weed Sci. Soc. Am., n.38, p.465, 1985.

MADHUN, Y.A., YOUNG, J.L., FREED, V.H. Binding of herbicides by water-soluble organic materials from soil. J. environ. qual., v.15, n.1, p.64-68, 1986.

MANGELS, G. Behavior of the imidazolinone herbicides in soil - A review of the literature. In: SHANER, D.L., O'CONNOR, S.L. (Eds.). The imidazolinone herbicides, Florida: CRC Press, Inc., 1991. p.191-209.

McKINNON, J., WEBER, J.B. Leaching and capillary movement of imazaquin in soil. Proc. So. Weed Sci. Soc. Am., n.41, p.337, 1988.

MONSANTO. Manual técnico de produtos. [s.n.]: São Paulo, 1990. 31p.

NICHOLLS, P.H. Factors influencing entry of pesticides into soil water. Pestic. sci., n. 22, p.123-137, 1988.

OGNER, G. Glyphosate application in forestecological aspects. II. The quality of water leached from forest soil lysimeters. Scand. j. for. res., n.2, p.469-480, 1987.

PICCOLO, A., CElANO, G., CONTE, P. Adsorption of glyphosate by humic substances. J. agric. food chem., v.44, n.8, p.2442-2446, 1996.

RODRIGUES, B.N., ALMEIDA, F.S. Guia de herbicidas. 3. ed. Londrina, PR, 1995. 675p.

SAEG. Sistema para Análises Estatísticas; versão 7.1. Viçosa: Fundação Arthur Bernardes, 1997.

SÁNCHEZ-MARTÍN, M.J., CRISANTO, T., ARIENZO, M. et al. Evaluation of the mobility of $\mathrm{C}^{14}$-labelled pesticides in soil by thin layer chromatography using a linear analyser. J. environ. sci. health, Part B, n.3, p.473-484, 1994.

SENESI, N., BRUNETTI, G., LA CAVA, P. et al. Adsorption of alachlor by humic acids from sewage sludge and amended and nonamended soils. Soil Sci., v.157, n.3, p.176184, 1994.

SPRANKLE, P., MEGGITT, W.F., PENNER, D. Rapid inactivation of glyphosate in the soil. Weed Sci., v.23, n.3, p.224-228, 1975a.

SPRANKLE, P., MEGGITT, W.F., PENNER, D. Adsorption, mobility, and microbial degradation of gl yphosate in the soil. Weed Sci., v.23, n.3, p.229-234, 1975b.

SUNDARAM, A., SUNDARAM, K.M.S. Solubility products of six metal-glyphosate complex in water and forestry soils, and their influence on glyphosate toxicity to 
plants. J. environ. sci. Health, Part B, n.4, p.583-598, 1997.

WEBER. J.B. Model soil systems, herbicide leaching and sorption. In: WILKINSON, R.E. (Ed.). Research methods in weed science. Atlanta: POP Enterprise, Inc., 1972. p.145-160.

WEBER, J.B., MILLER, C.T. Organic chemical movement over and through soil. In: SAWHNEY, B.L., BROWN, K. (Eds.). Reactions and movement of organic chemicals in soil, Madison, Wisconsin, USA: SSSA Inc., 1989. p.305-334 (SSSA Special Publication, 22).
WEBER. J.B., SWAIN, L.R., STREK, H.L. et al. Herbicide mobility in soil leaching columns. In: CAMPER, N.D. (Ed.). Research methods in weed science. 3 . ed., Atlanta: POP Enterprise, Inc., 1986. p.187217.

WEHTJE, G., DICKENS, R., WILCUT, J.W. et al. Sorption and mobility of sulfometuron and imazapyr in five Alabama soils. Weed Sci., v.35, p.858, 1987.

WINTON, K., WEBER, J.B. A review of field lysimeter studies to describe the environmental fate of pesticides. Weed Technol., n.10, p.202-209, 1996. 